\title{
„Fiktion streng realer Ereignisse und Fakten“" Tendenzen der literarischen Autofiktion von Fils (1977) bis Hoppe (2012)
}

\section{Jutta Weiser}

Das Titelzitat des vorliegenden Beitrags - im französischen Original heißt es „fiction d'événements et de faits strictement réels“ - stammt aus der bekannten ,Definition' der Autofiktion auf der hinteren Umschlagseite des Romans Fils (1977) von Serge Doubrovsky, der den Neologismus autofiction ins Leben gerufen hat. Gegenstand des Romans ist ein Tag aus dem Leben des Literaturprofessors ,Serge Doubrovsky', beginnend mit der Erinnerung an einen nächtlichen Traum gefolgt von der Fahrt zum Psychoanalytiker durch die Straßen New Yorks, der AnalyseSitzung, in der der Traum gedeutet wird, und schließlich einer Seminarsitzung über Racines Phèdre an der New York University. Wie in der Autobiographie weisen alle erzählten Ereignisse programmatisch einen authentischen Bezug zum Leben des Autors auf und sind von diesem sogar noch einmal peinlich genau auf ihre faktische Richtigkeit überprüft worden:

En bonne et scrupuleuse autobiographie, tous les faits et gestes du récit sont littéralement tirés de ma propre vie; lieux et dates ont été maniaquement vérifiés. La part d’invention dite romanesque se réduit à fournir le cadre et les circonstances d'une pseudojournée, qui serve de fourre-tout à la mémoire. (Doubrovsky 1988: 69) ${ }^{1}$

Wenngleich der Klappentext eine „Fiktion streng realer Ereignisse und Fakten“ ankündigt, wird Fils auf der Titelseite als ,Roman' deklariert und somit einem fiktionalen und nicht dem überwiegend faktualen Genre der Autobiographie ${ }^{2}$ subsumiert. Augenscheinlich verschmelzen faktuales und fiktionales Erzählen zu einer paradoxen Einheit; schließlich liegen das autobiographische Projekt und die paratextuelle Gattungszuordnung im Widerstreit. Zwar legt Doubrovsky selbst nahe, dass die Kennzeichnung als Roman möglicherweise nur eine Strategie sei, um die Autobiographie eines unbedeutenden Mannes an den Leser heranzutragen. ${ }^{3}$ Stimmte man dieser Erläuterung zu, so wäre die Autofiktion lediglich die

1 „Nach Art einer ordentlichen und gewissenhaften Autobiographie sind die dargestellten Fakten und Handlungen der Erzählung tatsächlich meinem eigenen Leben entnommen; Orte und Zeiten wurden sorgsam überprüft. Der Teil der romanesken Erfindung beschränkt sich darauf, den Rahmen und die Umstände eines Pseudo-Tags zu liefern, der dem Gedächtnis als Aufbewahrungsort dient". (Alle Übersetzungen sind von mir, J. W.)

2 Obgleich sich zweifellos in jede Autobiographie auch fiktive Elemente einschleichen, überwiegt hier - im Unterschied zum Roman ebenso wie zur Autofiktion - das Faktische. Vgl. dazu Schäfer (2008: 306).

3 Vgl. Doubrovsky (1988: 69): „La fiction serait donc ici une ruse du récit; n’étant pas de par son mérite un des ayants droit de l'autobiographie, l'homme quelconque' que je suis doit, pour capter le lecteur rétif, lui refiler sa vie réelle sous les espèces plus prestigieuses d'une 
Autobiographie der kleinen Leute, die sich in Auflagenhöhe und Verkaufszahlen, nicht aber in gattungstypologischer Hinsicht von den Bekenntnissen eines Aurelius Augustinus oder Jean-Jacques Rousseau unterschiede. Dennoch greifen der Bekanntheitsgrad des Autors in Kombination mit den verlegerischen Vermarktungsstrategien als Differenzierungskriterium zwischen Autobiographie und Autofiktion zu kurz. Doubrovsky selbst kommt es offensichtlich gerade auf die Unentscheidbarkeit zwischen dem autobiographischen und dem romanesken Diskurs an: „Ni autobiographie ni roman, donc, au sens strict, il [le texte] fonctionne dans l'entre-deux, en un renvoi incessant, en un lieu impossible et insaisissable ailleurs que dans l'opération du texte" (Doubrovsky 1988: 70). ${ }^{4}$

In der französischen Diskussion wird die autofiction bis heute weitgehend als literarisches Genre betrachtet, ${ }^{5}$ welches als „entre-deux“ eine Leerstelle zwischen dem Roman und der Autobiographie innerhalb des Gattungsmodells Philippe Lejeunes ausfüllt, insofern dem Leser sowohl ein pacte autobiographique (durch die Namensidentität von Autor und Ich-Erzähler) als auch ein pacte romanesque (durch die paratextuelle Gattungszuordnung) angeboten werden. ${ }^{6}$ Marie Darrieussecq hat in ihrem vielzitierten Artikel "L'autofiction: un genre pas sérieux" auf das Angebot beider Pakte seitens des Autors sowie das beständige Oszillieren zwischen dem Autobiographischen und dem Romanesken aufmerksam gemacht, welches aufgrund der „,fictionnalisation“ du factuel“ und der „,factualisation " du fictif“ einen „unentscheidbaren Text“ („texte indécidable“) hervorbringe (Darrieussecq 1996: 378).

In der hiesigen Frankoromanistik geht die Tendenz dahin, die Autofiktion nicht nur als neues hybrides Genre, sondern darüber hinausgehend auch als Provokation gängiger Gattungssysteme aufzufassen, so dass sie als eine Art Konterdiskurs zum herkömmlichen Textbegriff und den damit verbundenen Klassifizie-

existence imaginaire“./„Die Fiktion wäre hier also eine List der Erzählung. Da ich nicht zu denjenigen gehöre, die sich das Recht auf eine Autobiographie verdient haben, muss ich als ,gewöhnlicher Mensch', dem widerspenstigen Leser, um ihn für mich einzunehmen, mein wirkliches Leben in der glanzvolleren Gestalt eines erfundenen Lebens unterjubeln“.

„Er [der Text], der weder eine Autobiographie noch ein Roman ist im engeren Sinn, operiert in einem Zwischenbereich als ein unablässiges Verweisen an einem Ort, der außerhalb des Textgeschehens nicht möglich und nicht fassbar ist".

5 Jacques Lecarme spricht beispielsweise von einem „sous-genre romanesque“, einer Untergattung des Romans (Lecarme 2004: 14). In den jüngeren Publikationen fällt das Urteil in der Regel differenzierter aus; nach Genon bezeichnet der Terminus „tout autant un genre qu'une posture énonciative“/,ebenso eine Gattung wie auch eine Äußerungshaltung“(Genon 2013: 9).

6 Lejeune spricht von „cases aveugles“, (Leerstellen) in Bezug auf solche Fälle, in denen beispielsweise der Romanheld den Namen des Autors trägt, so dass hier zwar durch die Gattungszuordnung ein pacte romanesque geschlossen wird, während mit der Namensidentität ein Indikator für den pacte autobiographique vorliegt (vgl. Lejeune 1975: 31). Doubrovsky scheint von Lejeunes Feststellung, es gebe praktisch keinen Roman, in dem der Held mit dem Autor namensidentisch sei, inspiriert gewesen zu sein und bemerkt selbst in seinem Artikel „Autobiographie/vérité/psychanalyse“, dass Fils eine Leerstelle der Gattungssystematik fülle. Vgl. Doubrovsky (1988: 68). 
rungen fungiert. ${ }^{7}$ In diesem Sinne argumentiert Claudia Gronemann, dass sich Doubrovskys Konzept weder über rein formale Kriterien wie Namensidentität und paratextuelle Gattungsbezeichnung noch über den außertextuellen Befund eines ambivalenten Autor-Leser-Pakts erfassen lasse; ausschlaggebend seien in erster Linie die Sprachkritik, der zugrundeliegende Wahrheits- und Authentizitätsbegriff sowie das postmoderne Textverständnis, bei dem trotz aller Skepsis die Sinn- und Wahrheitssuche aufrecht erhalten bleibe (vgl. Gronemann 2002: 46-54).

Das Phänomen der Autofiktion erweist sich bei genauerer Betrachtung als wesentlich komplexer als es auf den ersten Blick erscheint und bedürfte einer entsprechend umfassenderen Darstellung, wollte man die unterschiedlichen Begriffsverwendungen und Konzeptualisierungen der letzten Jahrzehnte berücksichtigen, was ich an dieser Stelle ob der gebotenen Kürze nicht zu leisten vermag. ${ }^{8}$ Stattdessen möchte ich mich in einem close reading den programmatischen Sätzen auf dem Buchumschlag von Fils annähern, welche die wegweisende Konzeption Doubrovskys in nuce enthalten.

Im Anschluss daran werde ich - auch wenn dies hier nur skizzenhaft und anhand weniger ausgewählter Beispiele möglich ist - die Linien des daraus hervorgegangenen literarischen Diskurses bis zur Gegenwart umreißen. Ziel meiner Untersuchung ist somit zum einen die systematische Erfassung der Konzeption des Gründervaters der postmodernen Autofiktion und zum anderen ein summarischer Ausblick auf die aktuellen Tendenzen der Fiktionalisierung von Autorschaft und Subjekt.

\section{Was ist Autofiktion? - Versuch eines close reading der Buchrückseite von Fils}

Auf dem Buchumschlag des Romans Fils lesen wir Folgendes:

Autobiographie? Non, c'est le privilège réservé aux importants de ce monde, au soir de leur vie, et dans un beau style. Fiction d'événements et de faits strictement réels; si l'on veut autofiction, d'avoir confié le langage d'une aventure à l'aventure du langage, hors

7 So z. B. Gronemann (2013: 94): „Autofiktionen füllen keine ungenutzte Leerstelle zwischen Roman und Autobiographie, sie erneuern oder komplettieren den Kanon nicht, sondern setzen sich von der Gattungslogik gezielt ab. Die Autofiktion als Diskursmodell konzipiert die Beziehung zwischen Subjekt, Text und Medialität in kritischer Absetzung von repräsentationslogischen Traditionen neu".

8 In der französischen Literaturwissenschaft ist es allgemein üblich, zwischen einer engen und einer weiten Definition des Terminus ,Autofiktion' zu unterscheiden. Die enge Definition stammt von Serge Doubrovsky und beschränkt sich auf die Postmoderne der drei letzten Dezennien des 20. Jahrhunderts. Der weiten, a-historischen Definition von Gérard Genette, Vincent Colonna und Philippe Gasparini zufolge wäre jeder Text eine Autofiktion, der faktische und fiktive Elemente kombiniert und in dem ein autodiegetischer und mit dem Autor namensidentischer Erzähler vertreten ist. Demnach zählten z. B. auch Dantes Commedia oder Prousts Recherche zu den Autofiktionen. Zu den unterschiedlichen Positionen vgl. Kraus (2013: 74-103). 
sagesse et hors syntaxe du roman, traditionnel ou nouveau. Rencontres, fils de mots, allitérations, assonances, dissonances, écriture d'avant ou d'après littérature, concrète, comme on dit musique. Ou encore, autofriction, patiemment onaniste, qui espère faire maintenant partager son plaisir. (Doubrovsky 1977: Umschlag, Kursivierungen i. O.) ${ }^{9}$

Ich möchte mich Satz für Satz dieser häufig zitierten Passage annähern, die sich dem Leser sicher nicht in allen Teilen unmittelbar erschließt und daher als captatio lectoris an exponierter Stelle auf dem Buchumschlag eigentlich völlig ungeeignet scheint.

Autobiographie? Non, c'est le privilège réservé aux importants de ce monde, au soir de leur vie, et dans un beau style. - Zunächst wendet sich Doubrovsky dezidiert gegen die Zuordnung seines Textes zur Gattung der Autobiographie. Es handele sich dabei um ein Vorzugsrecht berühmter Persönlichkeiten, wohl nicht zuletzt auch deshalb, weil damit große Namen wie Augustinus, Rousseau und Goethe verbunden sind. In Anlehnung an Philippe Lejeune hebt Doubrovsky zwei für das autobiographische Schreiben charakteristische Merkmale hervor: das retrospektive Erzählen (die Autoren befinden sich „au soir de leur vie“) und ein gediegener Prosastil („un beau style"). ${ }^{10}$ Beide Charakteristika unterläuft Doubrovsky durch seine Art und Weise der Narration, in der erzählendes und erlebendes Ich weitgehend kongruent sind und der gefällige Prosastil durch assoziativ aneinandergereihte Wortfäden (fils de mots) substituiert wird.

Fiction d'événements et de faits strictement réels; si l'on veut autofiction, d'avoir confié le langage d'une aventure à l'aventure du langage, hors sagesse et hors syntaxe du roman, traditionnel ou nouveau. - Diese oxymorale Konstruktion aus Fiktionen und „streng realen Ereignissen und Fakten“ indiziert, dass die herkömmliche Polarität von Fakten und Fiktionen grundsätzlich unterlaufen wird. In der Tat entsprechen die dargestellten Ereignisse angeblich dem Faktischen, während die Form des Diskurses fiktiv sein soll. Wie kann die Form fiktiv sein, zumal damit doch vielmehr eine Angelegenheit der histoire als des discours bezeichnet wird?11 Eine

9 „Autobiographie? Nein, das ist ein Privileg der wichtigen Leute, an ihrem Lebensabend und in schönem Stil. Fiktion streng realer Ereignisse und Fakten, Autofiktion, wenn man will, die Sprache eines Abenteuers dem Abenteuer der Sprache überantwortet zu haben, außerhalb der Vernunft und der Syntax des traditionellen oder neuen Romans. Begegnungen, Wortfäden, Alliterationen, Assonanzen, Dissonanzen, vor- oder nachliterarische Schreibweisen, konkrete Literatur, so wie man Musik ausdrückt. Oder auch Autofriktion, geduldig onanistisch, die jetzt darauf hofft, ihre Lust zu teilen“.

10 Vgl. dazu die Definition von Lejeune (1975: 14): „Récit rétrospectif en prose qu'une personne réelle fait de sa propre existence, lorsqu'elle met l'accent sur sa vie individuelle, en particulier sur l'histoire de sa personnalité"./,Retrospektive Erzählung in Prosa, in der eine real existierende Person ihr eigenes Leben darstellt, sofern sie ihre individuelle Geschichte, insbesondere die Entwicklung ihrer Persönlichkeit fokussiert".

11 Dass diese Zuordnung unstimmig klingt, da die Erzählweise und die Reihenfolge der erzählten Fakten im Grunde keine Kriterien für die Fiktivität darstellen, hat auch Frank Zipfel angemerkt. Vgl. Zipfel 2009: 299: „Die nicht-chronologische psychoanalytischassoziative Anordnung der Ereignisse erscheint zwar statistisch gesehen eher untypisch für autobiographisches Schreiben, ist jedoch erzähllogisch im Hinblick auf die Fiktion unpro- 
Antwort auf diese Frage bietet die Psychoanalyse, die innerhalb des Konzepts der Autofiktion einen zentralen Stellenwert einnimmt und auf die ich im Anschluss noch einmal ausführlicher zurückkomme.

Mit dem Hinweis auf das ,Abenteuer der Sprache` werden die Signifikanten und das Sprachspiel zum Gegenstand des Schreibens erklärt. Insofern sich die Worte dem Schreiber entziehen und ein Eigenleben innerhalb des Textgewebes führen, steht die autofiction im Zeichen der Postmoderne. Es wird implizit eine neue Art des Romanschreibens angekündigt, die sich sowohl gegen den traditionellen Roman als auch gegen den nouveau roman abgrenzt. Dieses neue Schreiben konstituiert sich aus: Rencontres, fils de mots, allitérations, assonances, dissonances, écriture d'avant ou d'après littérature, concrète, comme on dit musique. - Mit diesen Worten kennzeichnet Doubrovsky die sprachliche Form seiner Autofiktion als ein Aufeinandertreffen (rencontres) und Zusammenlaufen der Wörter zu den titelgebenden Wortfäden, aus denen die Textur des Werkes mit Hilfe von Klangfiguren wie Alliterationen und Assonanzen gewoben wird. Die Rhetorik der phonetisch motivierten fils de mots findet sich im Roman Fils praktisch umgesetzt, sogar die ,Wortfäden' werden stellenweise durch die typographische Anordnung des Textes sichtbar. Bereits der zitierte Umschlagtext exponiert den klangassoziativen Mechanismus, wenn dem Signifikanten assonances im Syntagma die paronomastischen dissonances folgen, so dass ferner die Priorität der Form gegenüber dem Inhalt ebenso wie die Prädominanz des Signifikanten über das Signifikat sinnfällig werden.

Im Unterschied zu den Alliterationen und Assonanzen bezeichnet die Dissonanz kein sprachliches, sondern ein primär musikalisches Phänomen. Mittels der Paronomasie (assonances - dissonances) werden die semantischen Bereiche Literatur und Musik miteinander verknüpft. Doubrovsky kommt auf diese Weise zur „écriture concrète“ als Bezeichnung für seine eigene Schreibtechnik. Die Begrifflichkeit entlehnt er der von Pierre Schaeffer konzeptualisierten musique concrète, bei der Alltags- und Naturgeräusche auf Tonband gespeichert, anschließend elektronisch verfremdet und mit Klängen von Musikinstrumenten kombiniert und neu montiert werden. Die Klangumwandlung konkreter, realer Alltagsgeräusche in ein neu zusammengesetztes Kunstwerk kann somit als musikalisches Analogon der literarischen Autofiktion als einer diskursiven Neukombination ,realer Fakten' betrachtet werden. Ereignisse aus der eigenen Lebensgeschichte werden in einer Weise umgeformt, ausgedehnt, gekürzt oder verfremdet, dass daraus ein ästhetisches Konstrukt entsteht, das auf den Leser dieselbe Faszinationskraft ausübt wie ein Roman. ${ }^{12}$

blematisch, so dass auch auf der von Doubrovsky als relevant angegebenen Fiktionsebene des Erzählens keine wirkliche Fiktionalisierung (z. B. durch eine faktual nicht mögliche Perspektive) stattfindet."

12 Unter Berücksichtigung der Montagetechnik, der Verknüpfung von Kunst und Alltag sowie der an die écriture automatique erinnernden sprachspielerisch-assoziativen Diskursordnung werden auch die Affinitäten zum Surrealismus sinnfällig, der sich ebenso auf die fundamentalen Einsichten Sigmund Freuds stützt. 
Ou encore, autofriction, patiemment onaniste, qui espère faire maintenant partager son plaisir. - Eine weitere Paronomasie führt zum Begriff der autofriction, der ,Selbstreibung' als einer Art einsamer Selbstbefriedigung. Ziel der autof(r)iction ist es, die ,Lust am Text ${ }^{`}$ mit dem Leser zu teilen. Mit diesem Schlusssatz, der sicher nicht frei ist von Ironie und Selbstironie, wird die Autofiktion als narzisstische Selbstbespiegelung sinnfällig, was bis heute als Argument zum Zweck der Diffamierung des autofiktionalen Schreibens dient. Darüber hinaus demonstriert diese Formulierung aber auch noch einmal Doubrovskys Nähe zur Psychoanalyse: Der Narziss-Mythos ebenso wie die von Freud entwickelte Konzeption des Narzissmus sind wesentliche Grundlagen der Lacanschen Theorie des Spiegelstadiums. Dieses beinhaltet die Subjektkonstitution über das Imaginäre, welche mit der Unerreichbarkeit des Realen sowie mit der These, dass für die Selbsterkenntnis ein Anderer konstitutiv sei, einher geht (vgl. Lacan 1999).

\section{Autofiktion und Psychoanalyse: Das, Diktat des Realen ${ }^{\circ}$}

In der Tat ist die epistemologische Rückbindung an die Psychoanalyse, die in Fils nicht nur das thematische Zentrum bildet, sondern sich auch diskursiv in der assoziativen Struktur des Textes niederschlägt, ein entscheidendes, wenn nicht das entscheidende konstitutive Moment der Autofiktion Doubrovskys (vgl. Gronemann 1999: 245-248 und Weiser 2008). Die Verknüpfung der Strukturen des Unbewussten mit dem Sprachspiel geht dabei wesentlich auf die Psychosemiologie Jacques Lacans zurück. Dieser bedient sich in seinen Schriften und Seminaren einer Sprache, die ihren Gegenstand, die Unerschöpflichkeit der Strukturen des Unbewussten, unablässig nachbildet: Homophonien, Paronomasien und Polysemien bestimmen sowohl das Schreiben Lacans als auch dasjenige Doubrovskys. Es verwundert daher kaum, dass der Verfasser von Fils in Lacan eher einen Schriftsteller und Dichter sieht als einen Analytiker (vgl. Doubrovsky 1980: 170).

In Analogie zur Psychoanalyse werden im Schreibprozess Fiktionen entwickelt, welche die Annäherung an eine verschüttete Wahrheit ermöglichen. Dies geschieht im Wesentlichen über Assoziationen, Versprecher oder Fehlleistungen, wie sie Freud in seiner Psychopathologie des Alltagslebens analysiert hat. Die aventure du langage wird bei Doubrovsky zum Vehikel der Wiederentdeckung der eigenen Vergangenheit, so dass die gegenwärtige Schreibszene einen wichtigen Raum einnimmt. Die verdrängten und mit Hilfe des Schreibens erneut an die Oberfläche gelockten Geschehnisse aus dem Leben des Schreibers sind dabei stets von brisanter, wenn nicht traumatischer Natur (denn sonst wären sie - freudianisch gedacht - nicht in Vergessenheit geraten). Im Fall des jüdisch-stämmigen Doubrovsky betrifft dies vor allem das jüdische Schicksal während des Zweiten Weltkriegs.

Ich möchte ferner die These aufstellen, dass das autofiktionale Schreiben einem ,Diktat des Realen' gehorcht. Nach Lacan wäre das Reale dem Subjekt nicht unmittelbar zugänglich, da die libidinöse Ökonomie des Subjekts dem (zumeist trauma- 
tischen) Kern des Realen ausweicht. Das Reale wird auf Abstand gehalten und dennoch permanent umkreist, es kehrt beständig wieder und sucht das Subjekt heim; die Erzählung schnürt sich immer enger um den Kern (noyau) jenes ,traumatischen Dings', gegen das sich die psychische Abwehr richtet (vgl. Lacan 1973: 66).

Begreifen wir den autofiktionalen Text als Resultat eines ,Diktats des Realen', so folgt daraus zweierlei: Erstens scheint das Reale ungeachtet seiner prinzipiellen Inkommensurabilität punktuell, verschwommen und kontingent im Symbolischen der Sprache auf. Die sich dem Schreiber entziehende Eigendynamik und Spontaneität des Sprachspiels setzen die Mechanismen der Verdrängung und Diskursverknappung sporadisch außer Kraft. Insofern erfolgt eine durchaus programmatische Annäherung an die ,Wahrheit', d. h. an den traumatischen Kern. Mit dem Diskurs der Autofiktion, dem Rahmen einer (fiktiven) pseudo-journée, wird eine Syntax geschaffen, in die sich das aus den Sprachassoziationen generierte Unbewusste gewissermaßen wie in eine Schablone hineingießt. Die Autofiktion wäre demnach theoretisch ein schriftliches Äquivalent zur psychoanalytischen talking cure. Zweitens impliziert das ,Diktat des Realen', dass dem Schreiber (dem scripteur im Sinne Roland Barthes') die Verfügungsgewalt über den Text entzogen ist. Diese Einsicht, die mit dem poststrukturalistischen Diktum vom ,Tod des Autors' koinzidiert, hat sowohl handlungspragmatische als auch ethische Konsequenzen, insofern der von der Autorität des Schreibers gelöste, ,entfesselte‘ Diskurs mitunter ein retraumatisierendes Gefahrenpotential birgt, dem indes der schützende Rahmen einer institutionalisierten face-to-face-Therapie fehlt.

Wie sich diese fehlende Autorität über den Text in der Schreibpraxis manifestiert, lässt sich am Beispiel von Doubrovskys Le livre brisé (1989) veranschaulichen, dessen Textverlauf von einem einschneidenden Ereignis im Leben des Autors, dem Tod seiner 36jährigen Ehefrau, geprägt ist. Ilse war nicht nur die reale Ehefrau von Serge Doubrovsky, sondern sie ist als Leserin und Kommentatorin des ersten Teils „Absences“ zugleich eine zentrale Figur des Romans. ${ }^{13}$ Sie verfolgt die Entstehung des Buches, das auf schonungslose Weise Gewaltszenen aus ihrem Eheleben, ihre Schwangerschaftsabbrüche und ihre Depressionen bis zum versuchten Suizid thematisiert. Der Titel bezieht sich auf jene ,Bruchstelle` (brisure) im Roman, die durch Ilses Tod in der Lebenswirklichkeit und im Roman entstanden ist. Ilses Tod steht im Zentrum der letzten hundert Seiten unter der Kapitelüberschrift „Disparition“. Bei den Spekulationen über die bis zum Schluss ungeklärte Todesursache drängt sich unter anderem der Verdacht auf, dass Ilse eventuell durch ihre Lektüre eines besonders drastischen Kapitels über ihre Alkoholexzesse in den Tod getrieben wor-

13 Die Wirklichkeitsebenen lassen sich kaum noch differenzieren: Den geläufigen literaturwissenschaftlichen Kommunikationsmodellen entsprechend wäre Ilse als Romanfigur eine ,fiktive Leserin'; der Autor will uns aber glauben machen, dass sie zugleich ,reale Leserin“ (außerhalb des Textes) ist und dass ihr realer Tod auch denjenigen der Romanfigur impliziert. Aber gibt es hier überhaupt noch ein hors-texte? Die derridasche Antwort auf diese Frage ist hinlänglich bekannt und zählt zu den basalen poststrukturalistischen Devisen, denen Doubrovsky verpflichtet bleibt. 
den sei: „le chapitre „Beuveries‘ l'a liquidée, mon encre l'a empoisonnée, jeu de vérité parfois mortel [...], elle en est morte“ (Doubrovsky 1989: 391). ${ }^{14}$ Mit anderen Worten partizipieren das schreibende Subjekt und in diesem Fall auch die involvierte Leserin an einem gefährlichen, mitunter tödlichen Wahrheitsspiel.

\section{Autofiktion zwischen Sprachskepsis und Poststrukturalismus}

Es wurde bereits angedeutet, dass die Autofiktion im Zeichen einer prekären Epistemologie entstanden ist, welche in den 70er und 80er Jahren noch wesentlich vom Poststrukturalismus und der damit einhergehenden Problematisierung des Subjekts und der Autorschaft bestimmt ist. Die Infragestellung des Subjekts als Sinngebungsinstanz geht bekanntlich mit der linguistischen Trennung von Signifikant und Signifikat und einer grundlegenden Selbstreferentialität des sprachlichen Zeichens einher.

Die traditionelle, auf der idealistischen Vorstellung eines selbstmächtigen, autonomen Ichs basierende Autobiographie ist unter diesen veränderten Prämissen problematisch geworden. Die Substitution der Fakten durch die Fiktionen in der neuen Autobiographie und Autofiktion lässt sich im Rückgriff auf die Subjekttheorie erklären: Bei Lacan situiert sich das entfremdete, gespaltene Ich „dans une ligne de fiction" (Lacan 1999: 93). Auf dieser Basis wäre das kohärente retrospektive Erzählen eines autonomen, selbstgewissen Ichs unmöglich. Wäre die Autofiktion folglich als die postmoderne bzw. poststrukturalistische Autobiographie zu begreifen, ${ }^{15}$ deren autonome, selbstreferentielle Signifikanten kein Signifikat außerhalb des Textes besitzen und somit den bios notwendig dekonstruieren?

Dagegen ließe sich einwenden, dass Doubrovsky selbst dieser historischen Eingrenzung nicht unbedingt zustimmt, wenn er 1999 in einem Interview mit Bezug auf autofiktionale Texte wie Colettes La naissance du jour (1928), Jean Genets Journal du voleur (1949) und Louis-Ferdinand Célines D'un château l'autre (1957) bemerkt: ,je n’ai pas du tout inventé l'autofiction. J'ai inventé le nom, le mot. [...] si j’ai inventé le mot je n'ai absolument pas inventé la chose, qui a été pratiquée avant moi par de très grands écrivains" (Hughes 1999: unpaginiert). ${ }^{16}$

Wenn die Autofiktion demnach nicht notwendig an die postmoderne Epistemologie gekoppelt ist, so bleibt sie zumindest der modernen Sprachskepsis in der Nachfolge von Nietzsches Über Wabrheit und Lüge im außermoralischen Sinne (1873) verpflichtet. Ungeachtet des literaturwissenschaftlichen Konsens über die Post-

14 „das Kapitel ,Saufereien' hat sich erledigt, meine Tinte hat sie vergiftet, bisweilen tödliches Wahrheitsspiel [...], sie ist daran gestorben."

15 Arnaud Genon spricht in diesem Sinne von einer „renaissance postmoderne du genre autobiographique" (Genon 2013: 10).

16 „Ich habe die Autofiktion keineswegs erfunden. Ich habe den Namen erfunden, das Wort. [...] Zwar habe ich das Wort erfunden, jedoch mitnichten das Phänomen, das es vor mir schon gab und von sehr großen Schriftsteller praktiziert wurde." 
modernität der Autofiktion würde Doubrovsky selbst wohl eher zur Einschätzung der Autofiktion als eines modernen Phänomens tendieren, das sich historisch aus der Vorstellung einer autonomen, $d$. h. von der Intentionalität eines sprechenden oder schreibenden Subjekts gelösten Sprache herleitet, wie sie im ausgehenden 19. Jahrhundert in die Poetologie Mallarmés ebenso wie in die Psychoanalyse Freuds eingegangen ist. Vertreter des Poststrukturalismus wie Foucault, Derrida und Lacan haben die sprach- und subjektkritischen Konzepte der Philosophie Nietzsches, der Psychoanalyse und der modernen Lyrik fortgesetzt und radikalisiert. ${ }^{17}$ Doubrovskys Sprachspiele einschließlich der Typographie lassen sich sowohl in die Nähe der écriture Lacans als auch in die Nähe von Mallarmés Un coup de dés jamais n'abolira le hasard (1897) rücken. Die bekannte Devise céder l'initiative aux mots, mit der Mallarmé die Loslösung des Klangspiels von jedweder sprachlichen Referentialität postuliert, ${ }^{18}$ ist - wie Doubrovsky in einem Artikel mit dem anspielungsreichen Titel „l'initiative aux maux: écrire sa psychanalyse" argumentiert - nicht weit entfernt von Freuds Aufforderung an seine Patienten, alles auszusprechen, was ihnen soeben durch den Kopf geht. ${ }^{19}$

In Fils versucht Doubrovsky, aus den Fakten seiner Lebensgeschichte ein poetisches Syntagma zu kreieren, in dem sich die Signifikanten über phonetische Äquivalenzen miteinander verketten. Bemerkenswert ist dabei, dass die im Poststrukturalismus prekär gewordene sprachliche Referenz wieder in das Spiel der Signifikanten eingespeist wird:

17 Ferner ist zu berücksichtigen, dass die Infragestellung des idealistischen Subjekts in der französischen Philosophie nicht erst mit Foucault, Derrida und Lacan beginnt. Im Grunde wird eine grundlegende Skepsis gegenüber dem selbstmächtigen, verlässlichen Ich schon in der Moralistik des 16. und 17. Jahrhunderts (etwa bei Montaigne, La Rochefoucauld, Blaise Pascal oder Pierre Nicole) formuliert. Dieselbe Problematik schwingt in Rimbauds Je est un autre mit und verschärft sich später in der Psychoanalyse Lacans. Die philosophie- und subjektgeschichtliche Entwicklung in Frankreich (Moralistik, Skeptizismus, Cartesianismus sowie die Positionen von Sartre, Foucault und Lévinas im 20. Jahrhundert) steht - etwa im Unterschied zu Deutschland mit der Leibnizschen Monadologie sowie der Philosophie Kants, Fichtes, Hegels und Schellings - seit jeher im Zeichen eines zweifelhaften Subjektstatus. Auf die unterschiedlichen subjektgeschichtlichen Entwicklungen in Frankreich und Deutschland und deren Auswirkungen auf das autobiographisch-autofiktionale Schreiben habe ich bereits in einer früheren Publikation (Weiser 2008: 67) aufmerksam gemacht.

18 Vgl. Mallarmé (1945: 366): „L'œuvre pure implique la disparition élocutoire du poëte, qui cède l'initiative aux mots, par le heurt de leur inégalité mobilisés; ils s'allument de reflets réciproques comme une virtuelle traînée de feux sur des pierreries, remplaçant la respiration perceptible en l'ancien souffle lyrique ou la direction personnelle enthousiaste de la phrase“./„Das reine Werk erfordert das sprachgestalterische Verschwinden des Dichters, der den durch den Zusammenprall ihrer Unregelmäßigkeiten aktivierten Wörtern die Initiative überlässt. Sie entzünden sich an wechselseitigen Reflexen wie ein möglicher Feuerschweif auf Edelsteinen, wobei sie den spürbaren Atem der alten überschwänglichen Schöpferkraft oder die originelle, enthusiastische Lenkung des Satzes ersetzen“.

19 Vgl. Doubrovsky (1980: 165): „L',association libre', qui cède l'initiative orale aux mots, relève du même principe qui, sur le plan de l'initiative écrite, conduit à l'avènement du texte“./„Die ,freie Assoziation', die den Wörtern die mündliche Initiative überlässt, beruht auf demselben Prinzip, das im Bereich der Initiative des Schreibens zur Entstehung des Textes führt“. 
En écrivant Fils, j'ai eu toujours à l'esprit la précision documentaire, tout en laissant liberté entière au verbe. Je ne sais pas si je l'ai déjà dit, mais je sens que lorsque j’écris, je louche, parce que mon œil d'un côté regarde vers le référent - l'histoire qui m'est arrivée - et l'autre œil, en même temps, regarde le jeu des mots, la manière dont ils s'accouplent, s'assemblent etc. Et c'est à l'intérieur de ce jeu des mots que je glisse le référent. (Hughes 1999: unpaginiert) $^{20}$

In der hier beschriebenen Gratwanderung zwischen der referentiellen und der selbstreferentiellen Funktion des sprachlichen Zeichens gleiten die Fakten in ihrer primär referentiellen Funktion ins Innere des selbstreferentiellen Signifikantenspiels hinein, wobei der Schreiber bemüht ist, dokumentarische Präzision und lautmalerische Spracheffekte gleichzeitig umzusetzen. Die gelungene Koinzidenz des lebensweltlichen Faktums mit dem entsprechenden Sprachspiel scheint mithin den Kern der Poetologie Doubrovskys auszumachen.

Allerdings lässt sich ein derartiges Einspeisen der Fakten in die diskursive Fiktion des Sprachspiels weder mit Mallarmés Poetik der ,Entrealisierung noch mit der poststrukturalistischen Entreferentialisierung der sprachlichen Zeichen verrechnen. Strenggenommen erscheint das Faktische hier als Störfaktor, als ein Überbleibsel des überkommenen autobiographischen Schreibens, das aus einer früheren, prä-nietzscheanischen Epoche stammt. Die Unvereinbarkeit des Faktisch-Referentiellen mit dem postmodernen Verschwinden des Realen und der semiologischen Autoreferentialität scheint mir indes genau jener kruziale Punkt zu sein, auf den Doubrovsky die Aufmerksamkeit lenkt: die Unmöglichkeit des Autobiographischen im Zeitalter der Sprachskepsis und der prekären Referentialität.

Wenngleich das Biographische in der Autofiktion dekonstruiert wird, benötigt letztere die Tradition der Autobiographie als Folie. ${ }^{21}$ Mit Ansgar Nünning könnte man die skizzierte dekonstruktivistische Tendenz um einen konstruktiven Aspekt ergänzen, indem man die Autofiktionen „als Gattungsgedächtnis und Gattungskritik der Autobiographie“ (Nünning 2007: 270) anerkennt. Im Fall Doubrovskys wäre die Gattungskritik im Wesentlichen eine Subjektkritik, während das Gattungsgedächtnis in einer poetologischen Metareflexion besteht, die den Fokus auf die problematische Rekonstruktion der eigenen Geschichte, die Tücken des Schreibens und das Abenteuer der Sprache richtet. Diese Metarefle-

20 „Beim Schreiben von Fils hatte ich ständig die dokumentarische Präzision im Kopf und ließ dabei den Wörtern freien Lauf. Ich weiß nicht, ob ich das schon gesagt habe, aber ich habe beim Schreiben das Gefühl zu schielen, denn ein Auge blickt auf den Referenten die Geschichte, die ich erlebt habe - und das andere Auge blickt gleichzeitig auf das Spiel der Wörter, auf die Art und Weise, wie sie sich paaren, miteinander verbinden usw. Und ins Innere des Wortspiels lasse ich den Referenten einfließen“.

21 Vgl. dazu auch Gronemann (1999: 240). Frank Reiser gelangt in seiner Untersuchung der intertextuellen Bezugnahme Doubrovskys auf Sartres Les mots zu der Einsicht, dass in Le livre brisé zwar das Vorgängermodell würdigend angeeignet werde, aber dennoch eine kritische Distanzierung erfolge, da Sartres Modell „in seinen Verfahren und der Selbstpräsenz und -transparenz des Subjekts in der Retrospektive letztlich dem orthodoxen Rousseauschen Autobiografietypus verpflichtet und dadurch inauthentisch ist" (Reiser 2004: 222). 
xion birgt ein kritisch-dekonstruktives Potential, insofern sie die Paradoxien des Autobiographischen, d. h. die Kluft zwischen dem Leben (bios) und der Sprache/Schrift (graphia) aufdeckt und konsequent durch die fictio ersetzt. ${ }^{22}$ Autofiktion beinhaltet demnach auch eine Korrektur des Autobiographischen, insofern sie ein Bewusstsein schafft für die semiologische Schwierigkeit, wenn nicht Unmöglichkeit des Vorhabens, das eigene Leben zu verschriftlichen. Demgegenüber bleibt die Fiktion im Sinne einer literarischen Erfindung auf der Seite der Sprache. Das Ich schreibt sich in die Maschen des Textes ein, es existiert nicht unabhängig von der Sprache: „Pour l'autobiographe, comme pour n'importe quel écrivain, rien, pas même sa propre vie, n'existe avant son texte: Mais la vie de son texte, c'est sa vie dans son texte“ (Doubrovsky 1980: 188). ${ }^{23}$

\section{Zwischenfazit: Doubrovskys Konzept der Autofiktion}

Bevor ich mich der Autofiktion in der Nachfolge Doubrovskys zuwende, möchte ich an dieser Stelle noch einmal in gestraffter Form die Kriterien für das autofiktionale Schreiben festhalten, die sich aus dem bislang Erörterten herleiten:

1. Formal handelt es sich um ein Schreiben in der ersten Person, d. h. Erzähler und Protagonist bilden eine Personalunion, wobei

2. eine Namensidentität zwischen dem autodiegetischen Erzähler und dem Autor besteht.

3. Autofiktionen werden auf der Titelseite als Romane ausgewiesen.

4. Autofiktionen haben einen Bezug zur Gegenwart des Schreibens im Unterschied zur Retrospektive der Autobiographie.

5. Das Syntagma folgt keiner logisch-chronologischen, sondern einer assoziativen Ordnung (Stichwort: „fils de mots“/,aventure du langage“).

6. Autofiktionen basieren prinzipiell auf einer (post-)modernen, sprachskeptischen Epistemologie.

7. Insofern die Autofiktion zwischen dem Autobiographisch-Faktischen und der Romanfiktion oszilliert, ist ihr in gattungstypologischer Hinsicht eine grundlegende Ambiguität eingeschrieben. Die fiktiven Elemente werden in der traditionellen Autobiographie noch rhetorisch überspielt, in der Autofiktion hingegen bewusst ausgestellt.

8. Im Sinne eines ,Gattungsgedächtnisses ${ }^{\varsigma}$ und einer ,Gattungskritik ${ }^{\varsigma}$ enthält die Autofiktion metapoetologische Reflexionen auf die Problematik autobiogra-

22 In diesem Sinne bemerkt Gronemann (1999: 251): „Wahrhaftigkeit liegt hierbei in dem ,Versuch', die Inkompatibilität von Biographie und Text aufzudecken, anstatt sie durch syntaktische Kohärenz zu überdecken."

23 „Für den Autobiographen wie für jeden anderen Schriftsteller existiert nichts, nicht einmal sein eigenes Leben, vor dem Text. Vielmehr ist das Leben seines Textes sein Leben in seinem Text“. 
phischen Schreibens und schafft ein Bewusstsein für die Unvereinbarkeit von Leben und Sprache.

9. Im Sinne der Psychoanalyse kreisen Autofiktionen um einen traumatischen Kern, der punktuell und schemenhaft in der selbstreferentiellen Sprache aufscheint (Stichwort: ,Diktat des Realen') und sich der Verfügungsgewalt des Schreibers/Analysanden entzieht.

\section{Evidentia in narratione: Realitätseffekte in den Autofiktionen der 1990er und frühen 2000er Jahre}

In den 1990er Jahren werden die Interferenzen von Leben und Schreiben Teil einer multimedial angelegten rhetorischen Strategie, die darauf abzielt, Authentizität zu suggerieren und den Leser glauben zu machen, alles sei ,wahr und habe sich tatsächlich so zugetragen, wie es geschildert wird. Diese Tendenz führt zu einer generellen Popularisierung der Autofiktion und einem zunehmenden Abrücken vom literarisch-intellektuellen Höhenkamm, wie er in den 80er und den frühen 90er Jahren von der nouvelle autobiographie Robbe-Grillets und Nathalie Sarrautes repräsentiert wurde (vgl. Sarraute 1983; Robbe-Grillet 1985, 1987, 1994). Damit gerät das autofiktionale Schreiben in eine Reihe mit den Reality-Shows des Fernsehens und gibt den Lesern in Analogie zu den Live-Talkshows das Gefühl, unmittelbar an fremdem Leben teilzunehmen.

Die Strategie folgt einer Rhetorik des Vor-Augen-Stellens, der enargeia oder wie es bei Quintilian heißt - der evidentia in narratione, d. h. dass „etwas Wahres nicht nur ausgesprochen“, sondern „vorgeführt“ wird. ${ }^{24}$ Der Akzent verschiebt sich mithin von der psychoanalytischen Diegesis zur intermedialen Mimesis.

Um autofiktionale Realitätseffekte zu erzielen, greifen die Autoren auf unterschiedliche Mittel zurück, so z. B. auf die Nennung nachweislich real existierender Personen wie Schriftstellerkollegen, Fernsehmoderatoren, Verleger oder auch die Erwähnung authentischer Buchtitel (häufig der eigenen) im Roman; sie thematisieren den Schreibvorgang und den Literaturbetrieb und setzen überprüfbare Daten und Ereignisse aus dem eigenen Leben (d. h. solche Fakten, die mit dem allgemein bekannten biographischen Wissen und dem Wikipedia-Eintrag korrespondieren) bewusst ein oder rekurrieren auf politische, wirtschaftliche oder kulturelle Ereignisse, die aus der Tagespresse bekannt sind.

Teilweise gehen die auf diese Weise erzeugten Realitätseffekte mit einer entsprechenden Affekterregung einher, die wohl am stärksten durch tabuisierte und skandalverdächtige Themen hervorgerufen wird, welche allgemeine Betroffenheit

24 Vgl. Quintilianus (1995: 460-61, IV 2, 64): „evidentia in narratione, quantum ego intellego, est quidem magna virtus, cum quid veri non dicendum, sed quodammodo etiam ostendendum est, [...]“..„„Anschaulichkeit ist zwar, wenn ich recht sehe, in der Erzählung ein großer Vorzug, indem etwas Wahres nicht nur ausgesprochen, sondern gewissermaßen vorgeführt zu werden verdient, $[\ldots]$ “.. 
erzeugen (so z. B. Hervé Guiberts autofiktionale AIDS-Trilogie) oder eine voyeuristische Lust beim Leser auslösen, so z. B. pornographische Darstellungen, die bisweilen - gewissermaßen als exhibitionistisches Sahnehäubchen - von den meist weiblichen Autorinnen als die eigene sexuelle Praxis ausgegeben werden. Beispielhaft dafür ist die Literatur der sogenannten ,weiblichen Libertinage'. Diese erlebte um die Jahrtausendwende in Frankreich einen Boom und erreichte ihren Höhepunkt mit der Publikation der Romane Linceste (1999) von Christine Angot, La Nouvelle Pornographie (2000) von Marie Nimier und La vie sexuelle de Catherine M. (2001) von Catherine Millet. Hierzulande folgten dann mit etwas zeitlichem Verzug Charlotte Roches Verkaufsschlager Feuchtgebiete (2008) und Schoßgebete (2011).

Das Erscheinen derartiger Skandalromane wird in der Regel von einer hohen Medienpräsenz der jeweiligen Autorin begleitet, was im Hinblick auf den Authentizitätseffekt ebenso wie auf die damit einhergehenden ökonomischen Aspekte (Verkaufszahlen, Auflagenhöhe etc.) ein Übriges tut. So besteht Christine Angots intermediale Poetologie beispielsweise in einem gekonnten Wechselspiel zwischen skandalöser Buchpublikation (in Linceste enthüllt sie den sexuellen Missbrauch durch ihren Vater) und aufsehenerregendem Fernsehauftritt. Der Zuschauer beobachtet live am Bildschirm, wie Angot während Ardissons Talkshow Tout le monde en parle beleidigt das Fernsehstudio verlässt und kann in ihrem nächsten Buch ihre subjektive Schilderung des legendären Fernsehauftritts noch einmal nachlesen, bevor die Autorin dann ein weiteres Mal anlässlich der neuen Publikation in Ardissons Sendung zu sehen ist. ${ }^{25}$ Ganz im Sinne der Quintilianschen evidentia wird ,Wahres' hier nicht nur ausgesprochen, sondern auch vorgeführt.

Die Differenz zwischen Fiktion und Alltagswirklichkeit wird bei Angot ferner vollständig aufgehoben, insofern die Autorin Epitexte wie Interviews, Rezensionen oder Leserbriefe in ihre Romane integriert. Folglich lässt sich hier auch nicht mehr zwischen der ,Einschreibung' der Autorin in die Fiktion und der ,Fortschreibung ${ }^{6}$ der Autorfiktion durch Journalisten differenzieren. ${ }^{26}$ Die verblassende Grenze zwischen Fakten, Fiktionen und Inszenierungen geht mit der Durchlässigkeit von Text und medialem Kontext einher.

Rückkopplungseffekte zwischen dem Buch und der medialen Öffentlichkeit sind häufig von den Autoren (bzw. dem dahinterstehenden Kulturbetrieb) bewusst arrangiert und garantieren im Sinne des all is true in der Regel eine effektive

25 Zur Nivellierung der Grenze zwischen dem Werk und seinem medialen Kontext bei Angot vgl. Nickenig (2013) und Weiser (2013: 231-234).

26 Die Begrifflichkeit übernehme ich von Innokentij Kreknin, der zum einen zwischen ,Einschreibungen' der Autoren in einen Text und ,Fortschreibungen' dieses Textes durch andere Schreiber (Rezensenten, Kritiker, Verleger, Literaturwissenschaftler usw.) unterscheidet, zum anderen zwischen ,fiktionaler ${ }^{6}$ Ein- und Fortschreibung, d. h. in einem fiktionalen Genre wie z. B. dem Roman, und ,alltagswirklicher' Ein- und Fortschreibung in nichtfiktionalen Genres wie z. B. dem Interview (vgl. Kreknin 2014: 32-33). Autofiktionen zeichnen sich in der Regel dadurch aus, dass eine heuristische Unterscheidung von fiktionalen und alltagswirklichen Einschreibungen grundsätzlich erschwert bis unmöglich ist. 
Vermarktung. Dem Authentizitätseffekt wird mithin auch durch textexterne Faktoren, genauer gesagt durch den Medientrubel um ein Buch und die öffentliche bzw. mediale Präsenz des Autors in Interviews, Lesungen, Fernsehauftritten, im Social Network und auf der eigenen Website, Vorschub geleistet.

In den jüngeren Autofiktionen (etwa der letzten zehn Jahre) lässt sich - nicht nur in Frankreich - die zunehmende Tendenz eines Abrückens von den beschriebenen make believe-Strategien zugunsten einer prononcierten Ausstellung der Fiktionalität der Autorfigur im Text beobachten. Ich denke dabei an Texte wie Bret Easton Ellis' Lunar Park (2005), Michel Houellebecqs La carte et le territoire (2010) oder Felicitas Hoppes Hoppe (2012).

\section{Neue Formate der Autofiktion in den letzten zehn Jahren}

Wohl nicht zuletzt aufgrund der zunehmenden Kommerzialisierung und Popularisierung der ursprünglich intellektuell ausgerichteten Textsorte ist die Autofiktion in Frankreich in den letzten Jahren verstärkt in Misskredit geraten. Autofiktionen werden als seichte, (selbst)gefällige Texte oder auch als „écriture égolâtrique sans ambitions autres que narcissiques" (Burgelin 2010: 6) ${ }^{27}$ abqualifiziert. In der Tat lässt sich zu Beginn des neuen Jahrtausends eine solche antiklimaktische Entwicklung bilanzieren. Diese mag einerseits der grundlegenden Abkehr von der postmodern-poststrukturalistischen terreur théorique geschuldet sein, mit der dann auch das theoretische Fundament in Frage gestellt wurde, auf dem die autofiction Doubrovskys Ende der 1970er Jahre ebenso wie die nouvelle autobiographie Robbe-Grillets in den 1980er Jahren noch errichtet worden waren. Andererseits ist eine zunehmende Medialisierung und Intermedialisierung der Literatur im Zuge der Entstehung und Entwicklung des World Wide Web dafür verantwortlich. Das Internet ist nicht nur ein weiteres Medium neben Presse und Fernsehen, das die bereits skizzierte Vermarktung und Selbstvermarktung von Autoren und Büchern unterstützt, sondern es nimmt darüber hinaus sogar jedwede Hürde zur öffentlichen Selbstdarstellung: Jeder einzelne noch so unbedeutende Mensch hat die Möglichkeit, kostenfrei und ohne großes technisches Knowhow sein Leben in Wort und Bild einer weltweiten Öffentlichkeit zu präsentieren. Es ist unmöglich zu durchschauen, ob es sich bei Bloggern oder Usern eines Chatrooms um authentische oder erfundene Identitäten handelt. Auch hier ließe sich von ,Autofiktionen' sprechen, insofern sich digitale und digitalisierte Identitäten häufig aus wahren und erfundenen Elementen zusammensetzen.

Der Weblog als neues Format der Autofiktion zeichnet sich durch eine Mischung der Textformen aus: Er knüpft konzeptionell an die Form des Tagebuchs an, ferner an den Kommentar, die Glosse, den Leserbrief und andere journalistische Formate ebenso wie an den elektronischen Newsletter (vgl. Hallet: 2011: 95).

27 „selbstverherrlichende Schreibweise ohne andere als narzisstische Ambitionen“. 
Éric Chevillard schreibt seit September 2007 täglich drei kurze, aphoristische Textstücke in seinem Blog mit dem verheißungsvollen Titel L'autofictif und gewährt seinen Lesern damit Einblick in seine Schreibwerkstatt, denn nach Ablauf eines Jahres erscheinen die Einträge jeweils noch einmal in Buchform. Bislang sind sechs Bände bei dem kleinen Verlag Arbre Vengeur erschienen, die auf der Titelseite und im Untertitel der Gattung des Tagebuchs (Journal) zugeordnet werden. Aufschlussreich ist das Vorwort, in dem der Autor auch auf den Titel seines Blogs zu sprechen kommt:

En septembre 2007, sans autre intention que de me distraire d'un roman en cours d'écriture, j'ai ouvert un blog, quel vilain mot, j'ai donc ouvert un vilain blog et je lui ai donné un vilain titre, plutôt par dérision envers le genre complaisant de l'autofiction qui excite depuis longtemps ma mauvaise ironie. [...] Mon identité de diariste est ici fluctuante, trompeuse, protéiforme, raison pour laquelle je n'ai pas renoncé au titre d'origine, somme toute assez pertinent pour nommer cette entreprise. (Chevillard 2009: 7-8) ${ }^{28}$

Chevillards Blog L'autofictif steht mithin im Zeichen der Ironisierung und Persiflage der Autofiktion, die hier als eine bereits ausgelaufene literarische Moderichtung aufscheint.

Auch Chevillards Roman L'auteur et moi, der 2012 zur rentrée littéraire im Verlagshaus Minuit veröffentlicht wurde, scheint von Strukturen des Hypertextes inspiriert: Der Romantext besteht aus der an eine junge Frau gerichteten Rede eines Ich-Erzählers, der im Restaurant eine Mandelforelle bestellt und stattdessen einen Blumenkohlauflauf serviert bekommt. Die Erzählung dieser belanglosen Begebenheit wird um Fußnoten des auteur ergänzt, die u. a. auf Bekanntes aus der Biographie des realen Autors Éric Chevillard verweisen. Der Fußnotentext übernimmt eine Kommentarfunktion und hat damit einen metatextuellen und metafiktionalen Status. Die Identität der textinternen Autorfigur mit dem realen Autor wird zwar deutlich nahe gelegt; dies geschieht allerdings nicht über das Angebot eines ,autobiographischen Paktes' im Sinne Lejeunes, denn im Haupttext fehlt die Namensidentität von Autor, Erzähler und Figur, und der Fußnotentext des auteur ist in der dritten Person geschrieben. Beide Kriterien sprächen zusammen mit der paratextuellen Gattungsbestimmung als Roman für eine rein romaneske Fiktion, in die sich dennoch der ,Autor ${ }^{6}$ einschreibt. Dieser übernimmt zunächst die Funktion eines extradiegetischen Kommentators, um dann nach und nach ins Zentrum der Diegese vorzurücken. ${ }^{29}$

28 „Im September 2007 habe ich, nur um mich von einem in Arbeit befindlichen Roman abzulenken, einen Blog eröffnet, was für ein fieses Wort, ich habe also einen fiesen Blog eröffnet und ihm einen fiesen Titel gegeben, mehr aus Spott über das gefällige Genre der Autofiktion, das seit Langem meine bittere Ironie herausfordert. [...] Meine Identität als Tagebuchschreiber ist hier schwankend, betrügerisch, vielgestaltig; aus diesem Grund habe ich nicht auf den Originaltitel verzichtet, der dieses Unterfangen alles in allem ziemlich genau trifft“.

29 Der Fußnotentext ist insgesamt umfangreicher als der Haupttext; allein die Fußnote 26 erstreckt sich über 111 Seiten des insgesamt knapp 300 Seiten umfassenden Buches. 
In Hamburg erschien im gleichen Jahr der Roman Verteidigung der Missionarsstellung von Wolf Haas, der dem Leser eine Rohfassung seines Textes vorzustellen scheint, insofern die fragmentierte Romanfiktion von Notizen des ,Autors für eine spätere Überarbeitung des Textes unterbrochen wird. In eckigen Klammern lesen wir so z. B. „SPÄTER EINFÜGEN. NÄCHSTE WOCHE SCHNELL 50 SEITEN LEBENSGESCHICHTE ZUSAMMENSCHUSTERN“ (Haas 2012: 154) oder „LIEBER STREICHEN. NERVT“ (Haas 2012: 166). Ähnlich wie in Chevillards L'auteur et moi wird eine Autorfigur auf einer Metaebene in den Romantext eingelassen, die für die Komposition der Haupterzählung verantwortlich zeichnet.

Eine andere Form der Fiktionalisierung des Autors vollzieht Michel Houellebecq in seinem Roman La carte et le territoire (2010). Auch hier ist der Erzähler heterodiegetisch, dennoch taucht im Roman eine mit dem Autor namensidentische Figur auf, die nach dessen öffentlichem Image modelliert ist: ,Michel Houellebecq ' tritt als einsamer, melancholischer und etwas verschrobener Kauz auf den Plan mit Rotwein und Zigarette als unverzichtbare Markenzeichen. Neben der realistischen Modellierung der Autorfigur enthält der Roman weitere Fakten, z. B. die Erwähnung früherer Werke Houellebecqs oder das Auftreten real existierender Nebenfiguren wie des Schriftstellers Frédéric Beigbeder oder der Verlegerin Teresa Cremisi. ${ }^{30}$ Derartige Realitätseffekte kontrastieren mit der Romanhandlung, in der „Houellebecq' in einen Kriminalfall verstrickt wird, der in seiner bestialischen Ermordung, der anschließenden Zerstückelung seines Leichnams und dem Begräbnis in einem Kindersarg kulminiert. Dass es sich bei dem selbstironischen Plot um eine reine Fiktion handelt, lässt sich kaum bezweifeln. Oder vielleicht doch?

Im September 2011 - ein Jahr nach dem Erscheinen des Romans - berichteten die Medien von einem ,Verschwinden' Houellebecqs, nachdem dieser zu geplanten Lesungen anlässlich der niederländischen Übersetzung von La carte et le territoire nicht erschienen war. Es kamen Gerüchte über eine mögliche Ermordung auf, wie sie der Roman bereits antizipiert hat; ferner spekulierte man über eine Entführung durch Al-Qaida, bevor Houellebecq unvermittelt wieder in Paris auftauchte. Allem Anschein nach intendiert der Literatur- und Medienbetrieb, indem er dem Gerücht Vorschub leistet, eine Metalepse der Romanfiktion in die Lebenswirklichkeit. Das Gerücht über die Entführung des Schriftstellers inspirierte den Regisseur Guillaume Nicloux wiederum zu einer filmischen Doku-Fiktion, in der Michel Houellebecq selbst die Hauptrolle spielt. Es sollte ein fiktiver Rahmen geschaffen werden, innerhalb dessen sich nicht der öffentliche Autor Houellebecq, sondern der ,wahre' Michel Thomas (so sein bürgerlicher Name) entfalten könne. ${ }^{31}$ Aber auch

30 Zum Spiel mit den Realitätseffekten, das Houellebecq zum einen dazu dient, das überkommene Modell der Autofiktion zu persiflieren und zum anderen dazu, sein öffentliches Autorenimage zu korrigieren vgl. Ott (2013: 223-230) und Weiser (2013: 138-247).

31 So erklärt Nicloux in einem Interview am 27.08.2014: „En utilisant l'alibi fictionnel, je voulais aborder le documentaire par une fenêtre inhabituelle afin d'offrir une introspection houellebecquienne hors du commun... Prendre comme précepte que ,tout documentaire est une fictio' et utiliser le mensonge comme rampe de lancement pour mieux at- 
der Film L'enlèvement de Michel Houellebecq (2014), in dem Houellebecq seine eigene Rolle spielt, besiegelt das bekannte Autorenimage.

Ein ähnliches Spiel mit ihrem öffentlichen Autorenbild betreibt Felicitas Hoppe in ihrem Roman Hoppe (2012). - Hier besteht zwar eine geradewegs ins Auge springende Identität des Autornamens mit dem Buchtitel, gleichwohl handelt es sich um eine schon auf dem Buchdeckel als Roman ausgewiesene PseudoBiographie einer Erzählinstanz namens $f b$ über die Schriftstellerin Felicitas Hoppe. Also eine Auto-Biographie? Nein. Hoppe nimmt hier bewusst den Umweg über eine fiktionale Biographin, deren Initialen nicht zufällig mit denjenigen der realen Autorin und der Protagonistin der Roman-Biographie koinzidieren. Dennoch unterscheidet sich Hoppe von den Autofiktionen Doubrovskys durch eine pointierte Nicht-Identität von Erzählerin und Protagonistin, durch das Schreiben über jemand anderen in der dritten Person. Das Ich spaltet sich in zwei Instanzen: die Erzählerin $f h$ und die Figur ,Felicitas Hoppe', die natürlich beide nicht mit der Autorin Felicitas Hoppe zu verwechseln sind. Die Nähe erzeugende Namensidentität geht mit einer gleichzeitigen formalen Distanzierung einher, so dass hier eine absurde und hochironische Erzählsituation hergestellt wird. Inhaltlich wird im Roman die ,offizielle Biographie der Autorin dekonstruiert, indem der durch die Medien des Literaturbetriebs bekannten Version eine weitere Variante zur Seite gestellt wird, für die in diesem Fall indes Felicitas Hoppe mit ihrem Autornamen verantwortlich zeichnet und nicht der journalistische und massenmediale Betrieb.

Hoppe kehrt Doubrovskys Anspruch, „streng reale Ereignisse und Fakten“ in die autofiktionale Form einzubinden genau um, indem sie mittels fiktiver Fakten und Ereignisse zunächst den Leser verwirrt, der sich unabwendbar fragen wird, ob denn nun die im Buch zu lesende korrigierte Biographie, die zwar unter der Etikette ,Roman' firmiert, dafür aber aus der Feder Hoppes selbst stammt, oder die bekannte, u. a. auf Wikipedia verzeichnete, indes im Buch als ,falsch' deklarierte Autorenbiographie die richtige sei. Durch das Nebeneinander überprüfbarer Fakten - z. B. die mehrfache Erwähnung eigener, tatsächlich erschienener Bücher wie Picknick der Friseure oder Pigafetta - und Schilderungen nicht überprüfbarer Details - etwa der Hinweis auf das unveröffentlichte „Erstlingswerk“ Häsi, das Hasenkind (Hoppe 2012: 35) - stellt Hoppe jene Glaubwürdigkeit erzeugenden Strategien autofiktionalen Schreibens geradezu aus, indem sie erwiesene und nicht erwiesene Fakten nebeneinander stellt.

Die Konzentration auf das eigene Ich und das damit einhergehende Schreiben in der ersten Person, wie sie in den Autofiktionen in der Nachfolge Doubrovskys

teindre un espace de liberté qui n'a qu'un seul but, la captation d'instants de vérité“ (Cusseau 2014)./,Mit dem Alibi der Fiktion wollte ich das Dokumentarische von einer ungewöhnlichen Seite zur Sprache bringen, um eine ausgefallene Innensicht Houellebecqs zu bieten... den Grundsatz, dass ,alles Dokumentarische eine Fiktion ist' geltend machen und die Lüge als Abschussrampe nehmen, um mehr Freiräume zu erreichen, die nur ein einziges Ziel verfolgen: das Einfangen von Momenten der Wahrheit“. 
bis dato üblich waren, werden bei Hoppe von einer ironischen Verdopplungsstrategie abgelöst. Im Unterschied zu Doubrovskys sorgfältiger Überprüfung der Fakten, aber auch im Unterschied zur make-believe-Rhetorik der 1990er Jahre partizipiert der Leser von Hoppe bewusst an einem Spiel mit Fakten und Fiktionen. Das Ich spaltet sich in unterschiedliche Instanzen innerhalb der Narration, was eine Zersplitterung der ,Wahrheit' zur Folge hat. Bei alldem machen das Fabulieren einer neuen, alternativen Lebensgeschichte, die mit überprüfbaren Fakten bestückt ist, ebenso wie die Verwischung der Grenze zwischen Wahrheit und Fiktion den ästhetischen Reiz des Romans aus. Hier geht es nicht mehr - wie in der postmodernen Autofiktion des 20. Jahrhunderts - um ein Demonstrieren der sprachskeptischen Unmöglichkeit des (Auto-)Biographischen, sondern um ein Ausloten neuer Möglichkeiten fiktionaler Selbstentwürfe, die mit Doubrovskys Initialprojekt nicht mehr viel gemeinsam haben.

\section{Zusammenfassung: Aktuelle Tendenzen der Auto(r)fiktion}

Die unterschiedlichen Formen und Formate der Durchkreuzung von fiktionalem und faktualem Erzählen in der Gegenwartsliteratur verlangen nach neuen Maßstäben. Heutzutage muss sich autofiktionales Schreiben nicht mehr - wie dies noch für die postmoderne Autofiktion und die nouvelle autobiographie zutrifft durch eine Manifestation der Brüchigkeit des Subjekts und der sprachlichen Referentialität gegen die klassische Autobiographie im Stil Rousseaus oder Goethes abgrenzen. Unter dem Einfluss des Internets und der damit einhergehenden Medialisierung und Intermedialisierung der Erzählliteratur entwickeln die Autoren neue Modelle des literarischen Selbstentwurfs, für deren adäquate Analyse die Literatursemiotik allein nicht mehr ausreicht und darüber hinaus kultursoziologische und medienwissenschaftliche Parameter nötig sind. ${ }^{32}$

Ich möchte abschließend die oben beschriebenen gegenwärtigen Tendenzen zusammenfassen, wobei der nachfolgende Katalog keinen Anspruch auf Vollständigkeit erhebt und sich gewiss fortsetzen ließe.

1. In formaler Hinsicht löst die Tendenz zur Narration in der dritten Person den notwendig autodiegetischen Erzähler der Autobiographie und Autofiktion ab.

2. Daraus folgt die Aufhebung der für das autobiographische und autofiktionale Schreiben noch unerlässlichen (Namens-)Identität von Autor, Erzähler und Figur.

32 Vgl. zu dieser grundlegenden These Weiser/Ott (2013: 7-16) sowie die erkenntnisreiche Studie Kreknin (2014), in der die umfassenden Fiktionalisierungen von Autorschaft im Zusammenspiel der Medien (Romanfiktion, journalistische Epitexte, Weblog etc.) am Beispiel von Rainald Goetz, Joachim Lottmann und Alban Nikolai Herbst in einer Weise systematisiert werden, die eine Übertragung der heuristischen Leitgrößen auf ähnlich gelagerte Phänomene der Gegenwartskultur erlauben. 
3. Das Spiel der Signifikanten wird durch ein Spiel mit dem eigenen Autorenimage substituiert. An die Stelle der Selbstsuche in den Maschen eines Textes tritt die Selbstinszenierung in einer multimedialen Gesellschaft. Der Fokus richtet sich nicht mehr auf das Innenleben des schreibenden Subjekts, sondern auf die Außenwirkung des öffentlichen Autors. Statt von Autofiktion ließe sich demnach präziser von Autorfiktion sprechen.

4. Folglich tritt die Psychoanalyse als Leitdisziplin des autofiktionalen Schreibens hinter soziologischen, ökonomischen und medienwissenschaftlichen Aspekten zurück.

5. Aktuelle literarische Selbstdarstellungen sind häufig selbstironisch oder auch als Persiflagen des introspektiven Modells der Autofiktion und der écriture de soi angelegt.

6. Die mit faktischen, alltagsweltlichen Komponenten ausgestattete Auto(r)fiktion wird nicht nur durch die paratextuelle Gattungsbezeichnung ,Roman' als Fiktion ausgestellt, sondern häufig auch durch offenkundig erfundene Inhalte, so dass der Anspruch auf die Darstellung „streng realer Fakten“ obsolet wird. Dabei wird die Rhetorik der evidentia in narratione bisweilen ironisch unterlaufen.

7. Gegenwärtige Auto(r)fiktionen weisen eine Tendenz zur intertextuellen und intermedialen Metalepse auf, d. h. die Fiktionalisierung von Autorschaft geht über die Ränder des literarischen Textes hinaus, indem sie in Epitexten wie Interviews, Autorenporträts und Pressemeldungen ebenso wie in den Foren des Social Network und in anderen audiovisuellen Medien fortgeführt wird.

8. Daraus resultiert eine Zunahme der Rückkopplungseffekte und Metalepsen zwischen dem Text und seinem medialen Kontext, die auf eine Ununterscheidbarkeit von Fakten und Fiktion und eine prinzipielle Durchlässigkeit der Textgrenzen hinauslaufen. ${ }^{33}$

\section{Literatur}

Angot, Christine (1999) L'inceste. Paris: Stock.

Burgelin, Claude (2010) „Pour l'autofiction“. Autofiction(s). Hgg. Claude Burgelin, Isabelle Grell und Roger-Yves Roche. Lyon: Presses Univ. de Lyon. 5-21.

Céline, Louis-Ferdinand (1957) D'un château l'autre. Paris: Gallimard.

Chevillard, Éric (2009) L'autofictif (Journal 2007-2008). Talence: Arbre Vengeur.

- (2012) L'auteur et moi. Paris: Minuit.

33 So behauptet etwa Thomas Glavinic in seinem Roman Das bin doch ich, er habe den Wikipedia-Eintrag zu seiner Person selbst angelegt, wobei er absichtlich ein falsches Geburtsdatum angegeben und einen seiner Romane negativ bewertet habe (vgl. Glavinic 2007: 21112). Diese Information wurde von anderen Wikipedia-Autoren anhand der Genese des Eintrags verifiziert und als wahrscheinlich deklariert. Vgl. <http://de.wikipedia.org/wiki/ Thomas_Glavinic\#Thomas_Glavinic_und_Wikipedia > (Stand: 4.8.2014). 
Colette (1928) La naissance du jour. Paris: Flammarion.

Colonna, Vincent (2004) Autofiction et autres mythomanies littéraires. Auch: Tristram.

Cusseau, Clément „L'Enlèvement de Michel Houellebecq: nos questions au réalisateur Guillaume Nicloux“ Interview du 27/08/2014. <http://www.allocine. fr/article/fichearticle_gen_carticle=18636264.html $>$ (letzter Zugriff: 21. Sept. 2014).

Darrieussecq, Marie (1996) „L’autofiction: un genre pas sérieux“. Poétique 27: 36780.

Doubrovsky, Serge (1977) Fils. Paris: Galilée.

- (1980) „L'initiative aux maux: écrire sa psychanalyse“. Parcours critique. Paris: Galilée. 165-201.

- (1988) „Autobiographie/Vérité/Psychanalyse“. Autobiographiques: de Corneille à Sartre. Paris: Presses Univ. de France. 61-79.

- (1989) Le Livre brisé. Paris: Grasset.

Ellis, Bret Easton (2005) Lunar Park. London: Picador.

Freud, Sigmund (1968) Gesammelte Werke 4: Zur Psychopathologie des Alltagslebens. Frankfurt a. M.: Fischer.

Gasparini, Philippe (2004) Est-il je?: roman autobiographique et autofiction. Paris: Seuil. - (2008) Autofiction: une aventure du langage. Paris: Seuil.

Genet, Jean (1949) Le journal du voleur. Paris: Gallimard.

Genette, Gérard (1991) Fiction et diction. Paris: Seuil.

Genon, Arnaud (2013) Autofiction: pratiques et théories. Articles. Paris: Mon Petit Éditeur.

Glavinic, Thomas (2007) Das bin doch ich. München: Hanser.

Gronemann, Claudia (1999) „,Autofiction“ und das Ich in der Signifikantenkette. Zur literarischen Konstitution des autobiographischen Subjekts bei Serge Doubrovsky“. Poetica 31: 237-62.

- (2002) Postmoderne/Postkoloniale Konzepte der Autobiographie in der französischen und maghrebinischen Literatur. Hildesheim: Olms.

- (2013) „lui dire que j'étais [...] un homme comme lui': Autofi(c)ktionales intermediales Schreiben bei Abdellah Tä̈a“. Autofiktion und Medienrealität: Kulturelle Formungen des postmodernen Subjekts. Hgg. Jutta Weiser und Christine Ott. Heidelberg: Winter. 91-106.

Guibert, Hervé (1990) A l'ami qui ne m’a pas sauvé la vie. Paris: Gallimard.

- (1991) Le protocole compassionel. Paris: Gallimard.

- (1992) L'bomme au chapeau rouge. Paris: Gallimard.

Hallet, Wolfgang (2011) „Medialisierung von Genres am Beispiel des Blogs und des multimodalen Romans: Von der Schrift-Kunst zum multimodalen Design". Medialisierung des Erzäblens im englischsprachigen Roman der Gegenwart: Theoretischer Bezugsrahmen, Genres und Modellinterpretationen. Hgg. Ansgar Nünning und Jan Rupp. Trier: WVT. 85-116. 
Haas, Wolf (2012) Verteidigung der Missionarsstellung. Hamburg: Hoffmann und Campe.

Hoppe, Felicitas (2012) Hoppe. Frankfurt a. M.: Fischer.

Houellebecq, Michel (2010) La carte et le territoire. Paris: Flammarion.

Hughes, Alex (1999) „Entretien avec Serge Doubrovsky, à l'occasion de la parution de ,Laissé pour conte', en janvier 1999“. <https://www.yumpu.com/fr/ document/view/19263341/iii-3-1-portrait-des-personnages-de-la-promesse-delaube/151> (letzter Zugriff: 10. Aug. 2014).

Kraus, Esther (2013) Faktualität und Fiktionalität in autobiographischen Texten des 20. Jabrbunderts. Marburg: Tectum.

Kreknin, Innokentij (2014) Poetiken des Selbst: Identität, Autorschaft und Autofiktion am Beispiel von Rainald Goetz, Joachim Lottmann und Alban Nikolai Herbst. Berlin: De Gruyter.

Lacan, Jacques (1973) Le Séminaire, livre XI (Les quatre concepts fondamentaux de la psychanalyse). Paris: Seuil.

- (1999) „Le stade du miroir comme formateur de la fonction du Je telle qu'elle nous est révélée dans l'expérience psychanalytique“. Écrits I: Nouvelle édition. Paris: Seuil. 92-99.

Lecarme, Jacques (2004) „Origine et évolution de la notion d'autofiction“. Le roman français au tournant du XXIe siècle. Hgg. Bruno Blanckeman, Aline MuraBrunel und Marc Dambre. Paris: Presses Sorbonne Nouvelle. 13-23.

Lejeune, Philippe (1975) Le pacte autobiographique. Paris: Seuil.

Mallarmé, Stéphane (1945) „Variations sur un sujet“. Evres complètes. Hgg. Henri Mondor und G. Jean-Aubry. Paris: Gallimard. 355-420.

- (1945) „Un coup de dés“. Evres complètes. Hgg. Henri Mondor und G. JeanAubry. Paris: Gallimard. 455-77.

Millet, Catherine (2001) La vie sexuelle de Catherine M. Paris: Seuil.

Nickenig, Annika (2013) „Mediale Transgressionen. Intertextualität und Aneignung in Christine Angots Autofiktionen“. Autofiktion und Medienrealität. Kulturelle Formungen des postmodernen Subjekts. Hgg. Jutta Weiser und Christine Ott. Heidelberg: Winter. 129-50.

Nicloux, Guillaume (2014) L'enlèvement de Michel Houellebecq (Film). Frankreich: Le pacte.

Nietzsche, Friedrich (1919) Werke 2: Über Wabrbeit und Lüge im außermoralischen Sinne / Unzeitgemäße Betrachtungen. Stuttgart: Kröner.

Nimier, Marie (2000) La nouvelle pornographie. Paris: Gallimard.

Nünning, Ansgar (2007) „Metaautobiographien: Gattungsgedächtnis, Gattungskritik und Funktion selbstreflexiver fiktionaler Autofiktionen“. Autobiographisches Schreiben in der deutschsprachigen Gegenwartsliteratur. Band 2. Hgg. Christoph Parry und Edgar Platen. München: Iudicium. 269-92.

Ott, Christine (2013) „Literatur und die Sehnsucht nach Realität. Autofiktion und Medienreflexion bei Michel Houellebecq, Walter Siti und Giulio Ming- 
hini". Autofiktion und Medienrealität: Kulturelle Formungen des postmodernen Subjekts. Hgg. Jutta Weiser und Christine Ott. Heidelberg: Winter. 209-31.

Quintilianus, Marcus Fabius (1995) Institutionis Oratoriae / Ausbildung des Redners [1972]. 2 Bde. Hg. Helmut Rahn. Darmstadt: Wissenschaftliche Buchgesellschaft.

Reiser, Frank (2004) „Autobiografie an der Grenze postmoderner Praxis: Serge Doubrovsky“. PostModerne De/Konstruktionen: Etbik, Politik und Kultur am Ende einer Epoche. Hg. Susanne Kollmann. Münster: LIT. 215-27.

Robbe-Grillet, Alain (1985) Le miroir qui revient. Paris: Minuit.

- (1987) Angélique ou l'enchantement. Paris: Minuit.

- (1994) Les derniers jours de Corinthe. Paris: Minuit.

Roche, Charlotte (2008) Feuchtgebiete. Köln: Dumont.

- (2011) Schoßgebete. München: Pieper.

Sarraute, Nathalie (1983) Enfance. Paris: Gallimard.

Schäfer, Christina (2008) „Die Autofiktion zwischen Fakt und Fiktion“. Im Zeichen der Fiktion: Aspekte fiktionaler Rede aus historischer und systematischer Sicht. Hgg. Irina O. Rajewsky und Ulrike Schneider. Stuttgart: Steiner. 299-326.

Weiser, Jutta (2008) „Psychoanalyse und Autofiktion“. Literaturtheorie und sciences bumaines: Frankreichs Beitrag zur Methodik der Literaturwissenschaft. Hg. Rainer Zaiser. Berlin: Frank \& Timme. 43-67.

- (2013) „Der Autor im Kulturbetrieb: Literarisches Self-Fashioning zwischen Selbstvermarktung und Vermarktungsreflexion (Christine Angot, Frédéric Beigbeder, Michel Houellebecq)“. Zeitschrift für französische Sprache und Literatur 123.4: 225-50.

Weiser, Jutta, und Christine Ott (2013) Hgg. Autofiktion und Medienrealität: Kulturelle Formungen des postmodernen Subjekts. Heidelberg: Winter.

Zipfel, Frank (2009) „Autofiktion. Zwischen den Grenzen von Faktualität, Fiktionalität und Literarität?““. Grenzen der Literatur: Zu Begriff und Phänomen des Literarischen. Hg. Simone Winko. Berlin: De Gruyter. 285-314. 